\title{
Investigation on Electromagnetic Field Characteristics of Interior Permanent Magnet Synchronous Machine Considering Harmonics of Phase Current due to Influence of Mechanical Energy Storage System
}

\author{
Yu-Seop Park* \\ Department of Electrical Engineering, Korea National University of Transportation, Chungju 27469, Korea
}

(Received 17 February 2017, Received in final form 13 March 2017, Accepted 15 March 2017)

\begin{abstract}
This paper investigates the influence of mechanical energy storage on the interior permanent magnet synchronous machine (IPMSM) when it is operated in the generating mode. An IPMSM with six-poles and nineslots employing concentrated coil winding type is considered as the analysis model, and a surface-mounted permanent magnet synchronous motor directly connected to a heavy wheel is applied as the mechanical energy storage system by using the moment of inertia. Based on the constructed experimental set-up with manufactured machines and power converters, the generated electrical energy is converted into the mechanical energy, and the electromagnetic filed characteristics of IPMSM are subsequently investigated by applying the measured phase current of IPMSM based on finite element method. Compared to the characteristics in a noload condition, it is confirmed that the magnetic behavior, radial force, and power loss characteristics are highly influenced by the harmonics of the phase current due to the mechanical energy storage system.
\end{abstract}

Keywords : IPMSM, MESS, power converter

\section{Introduction}

Recently, owing to its higher energy density and superior performance as compared to other motor types [1], the interior permanent magnet synchronous motor (IPMSM) has been widely employed in various industrial applications. As it is very well-known, since the motor type uses magnetic torque as well as reluctance torque, its high torque characteristic per volume resulting in satisfying efficiency. In addition, by its flux weakening control, the operating speed range can be wide. Therefore, for the electrical and hybrid vehicle systems, this machine type is very popular, so relevant studies have been very widely performed.

On the other hand, according to its applications, the motor can be operated under motoring mode and generating mode. So far, efforts to improve the performance of the machine in the motoring mode, such as the reduction of cogging torque and torque ripple [2], the minimization of power loss [3] and harmonic distortion of the back electromotive force [4] have been carried out in previous

(C)The Korean Magnetics Society. All rights reserved.

*Corresponding author: Tel: +82-43-841-5148

Fax: +82-43-841-5140, e-mail: yspark@ut.ac.kr studies. On the other hand, when the machine is operated in the generating mode, the current characteristics are entirely different from those in the motoring mode due to the influence of power converters, which can have a significant influence on the performance of the machine as previous studies have been reported the relevant characteristics. In [5], the authors dealt with the rotor losses due to rectifiers in their high speed synchronous generator, and conducting shield was employed. In [6], segmented magnets are introduced for eddy current reduction resulted from the rectifiers while [7] presented that the 5th harmonic component has high influence on rotor loss. Therefore, a related study is highly required for stable system operation; hence, in this study, an experimental set-up with an IPMSM in the generating mode is constructed with experimental set-up, and its relevant electromagnetic field characteristics are investigated based on the measured current while the generated electrical power is stored in a mechanical energy storage system (MESS), which consists of a heavy wheel and a surfacemounted permanent magnet synchronous motor (SPMSM). Using the results obtained in this study, the influence of the harmonics of the phase current due to the energy storage on the IPMSM is investigated. 


\section{Construction of Mechanical Energy Storage System in Generating Mode of IPMSM}

\subsection{Manufactured Machines}

The IPMSM investigated in this paper is presented in Fig. 1. As shown in the figure, the machine has six poles with nine slots, and a concentrated coil winding is applied in the stator core while the rotor employs rare-earth PMs for high energy density. Based on the finite element method (FEM), its electromagnetic field characteristics are investi- gated by applying the measured current obtained from the constructed experimental set-up. The design specifications are summarized in Table 1. On the other hand, in order to realize the MESS, another motor, which is the SPMSM, is employed in this research. The manufactured motor is presented in Fig. 2, and its machine size is briefly presented in Table 2. In this study, the SPMSM is directly connected to a heavy wheel to utilize the moment of inertia while the MESS is operated. Since this paper deals with the influence of the mechanical energy storage, its generating mode is not specifically addressed.
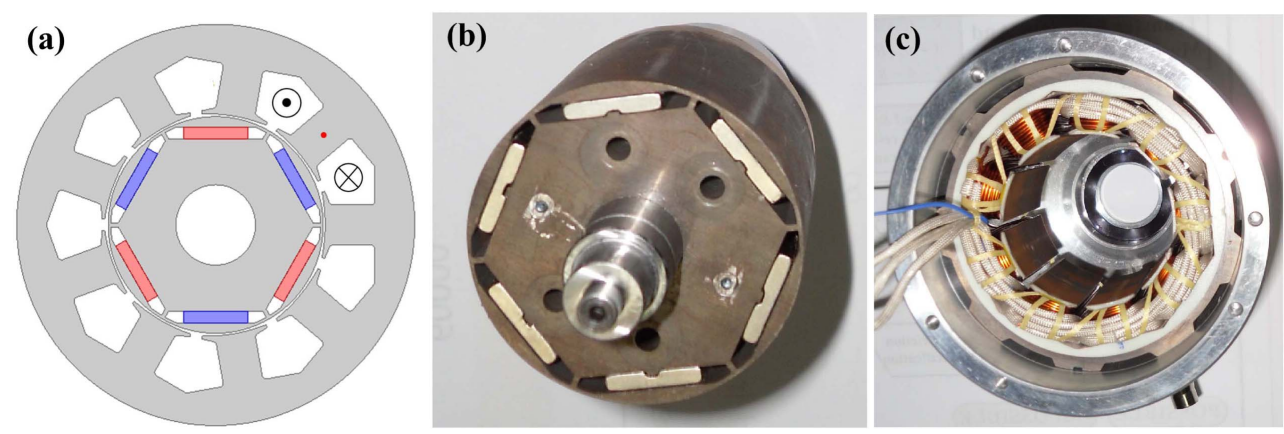

Fig. 1. (Color online) Analysis model of IPMSM and manufactured machine: (a) magnetic structure, (b) rotor with inserted PMs, (c) stator with coil winding and housing.

Table 1. Design Specification of IPMSM.

\begin{tabular}{cccc}
\hline \hline Specification & Value & Specification & Value \\
\hline Number of Poles & 6 & Number of Coil Turns & $84($ turns $)$ \\
Number of Slots & 9 & Parallel Branch & 1 \\
Outer Dia. of Stator & $100(\mathrm{~mm})$ & Resistance & $2.5(\mathrm{ohm})$ \\
Outer Dia. of Rotor & $54(\mathrm{~mm})$ & Voltage Constant & $22.7(\mathrm{~V} / 1 \mathrm{krpm})$ \\
Inner Dia. of Stator & $55(\mathrm{~mm})$ & Rated Power & $230(\mathrm{~W})$ \\
Inner Dia. of Rotor & $20(\mathrm{~mm})$ & Rated Torque & $1.1(\mathrm{Nm})$ \\
Stack Length & $55(\mathrm{~mm})$ & Rated Current & $1.5(\mathrm{~A})$ \\
Br of PM & $1.25(\mathrm{~T})$ & Maximum Speed & $6,000(\mathrm{rpm})$ \\
\hline
\end{tabular}
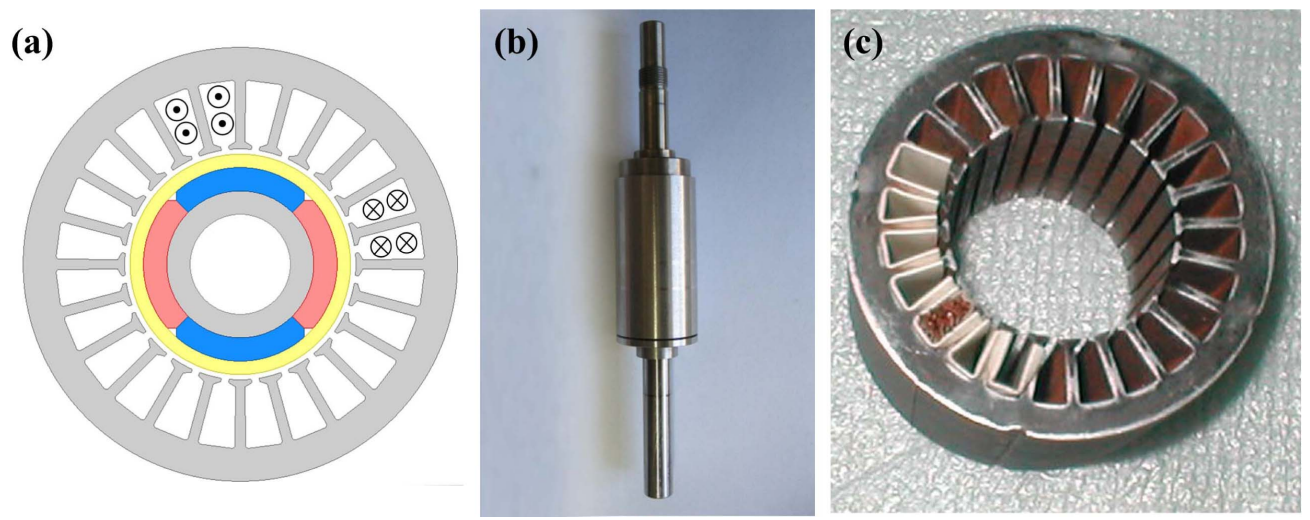

Fig. 2. (Color online) SPMSM employed in mechanical energy storage system : (a) magnetic structure, (b) rotor with sleeve, (c) stator core. 
Table 2. Design Specification of SPMSM.

\begin{tabular}{cccc}
\hline \hline Specification & Value & Specification & Value \\
\hline Number of Poles & 4 & Resistance & $0.24(\mathrm{ohm})$ \\
Number of Slots & 24 & Voltage Constant & $3.7(\mathrm{~V} / 1 \mathrm{krpm})$ \\
Outer Dia. of Stator & $65(\mathrm{~mm})$ & Rated Power & $200(\mathrm{~W})$ \\
Outer Dia. of Rotor & $34.6(\mathrm{~mm})$ & Rated Torque & $0.96(\mathrm{Nm})$ \\
Inner Dia. of Stator & $33(\mathrm{~mm})$ & Rated Current & $0.5(\mathrm{~A})$ \\
Inner Dia. of Rotor & $15(\mathrm{~mm})$ & Maximum Speed & $2,000(\mathrm{rpm})$ \\
Stack Length & $48(\mathrm{~mm})$ & Dia. of Wheel & $125(\mathrm{~mm})$ \\
Br of PM & $1.04(\mathrm{~T})$ & Height of Wheel & $50(\mathrm{~mm})$ \\
Number of Coil Turns & $112($ turns $)$ & Weigh of Wheel & $18.3(\mathrm{~kg})$ \\
Parallel Branch & 2 & Material of Wheel & STS630
\end{tabular}

\subsection{Construction of Experimental Set-up}

An experimental set-up was constructed to investigate the influence of the mechanical energy storage on the performance of the IPMSM, and its construction is briefly illustrated in Fig. 3. As shown at the bottom left of the figure, an induction motor is directly connected to the IPMSM to provide the artificial mechanical input power, whereas the IPMSM is integrated with an AC-DC-DC converter. The AC power, which is generated by the IPMSM, is converted to DC power by the rectifier included in the converter. The DC link voltage is also connected to a space vector pulse width modulation (SVPWM) inverter, and the MESS, which consists of the SPMSM and the heavy wheel, is operated by the inverter. When the MESS is not operated, the IPMSM is under the noload condition with negligible phase current from a systemic perspective. On the other hand, when the MESS starts rotating, which means that the mechanical energy is being stored, the inverse torque is subsequently generated with the increased phase current value in the IPMSM. The experimental set-up with the manufactured motors

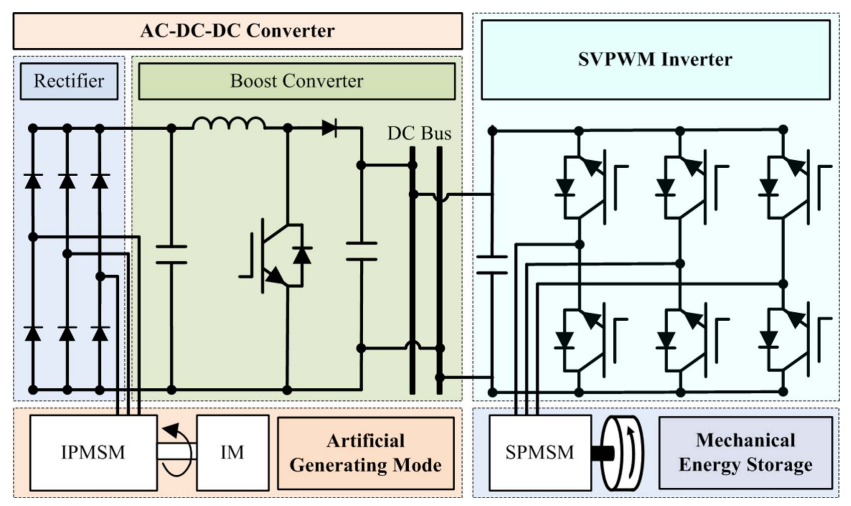

Fig. 3. (Color online) Structure of IPMSM system in generating mode with power converters to store mechanical energy through a heavy wheel.

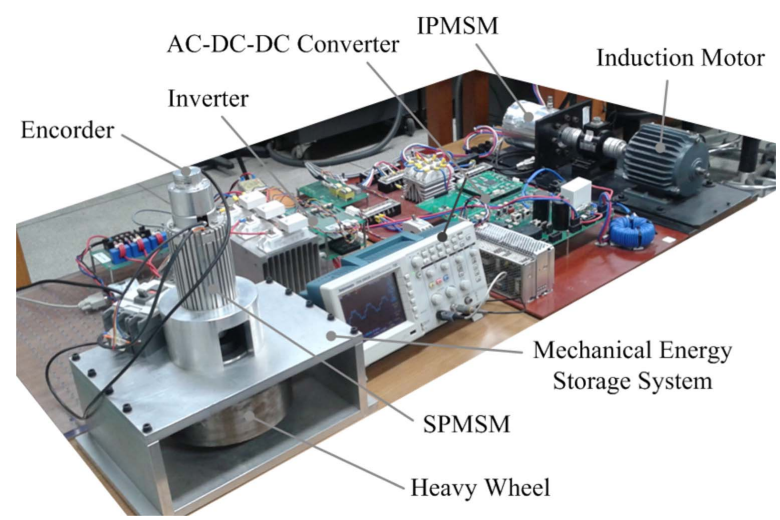

Fig. 4. (Color online) Constructed experimental set-up: IPMSM in generating mode connected to mechanical energy storage with power converters.

and power converters is presented in Fig. 4, and the phase current in the IPMSM is measured using a current sensors.

\subsection{Machine Characteristics according to Operating} Mode

Although this paper does not address the design procedure of the IPMSM, since FEM is employed to analyze the performance of the machine, the analysis method should be validated. At a rotating speed of 1,175(rpm), Fig. 5 presents the analyzed phase and line-to-line voltage, and flux linkage; further, it is confirmed that obtained value of the line-to-line voltage is consistent with the measured values presented in Fig. 6. In other words, the analyzed maximum value of the line-to-line induced voltage is $38.6(\mathrm{~V})$, while the measured value is $39.0(\mathrm{~V})$. The minor difference results from unpredictable manufacturing processes.

On the other hand, in load condition, the electromagnetic field analysis is also performed according to operating mode, which are the motoring mode and generating mode. In Fig. 7, the torque characteristic according to the 


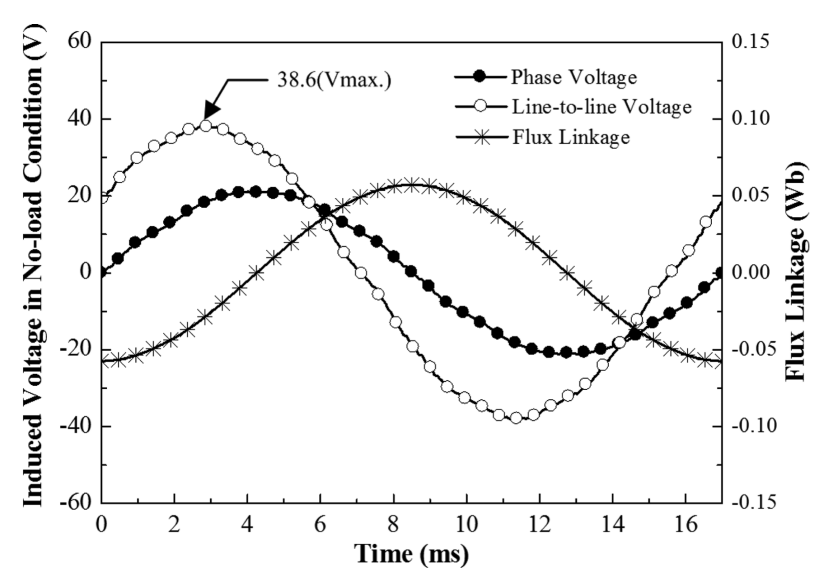

Fig. 5. Analyzed phase and line-to-line induced voltage with flux linkage of IPMSM in no-load condition (1,175 rpm).

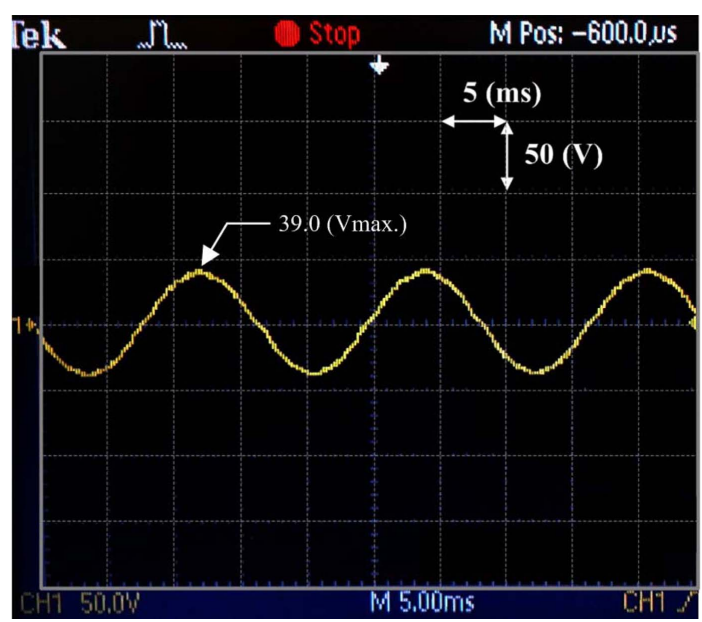

Fig. 6. (Color online) Experimentally measured line-to-line induced voltage of IPMSM in no-load condition (1,175 rpm).

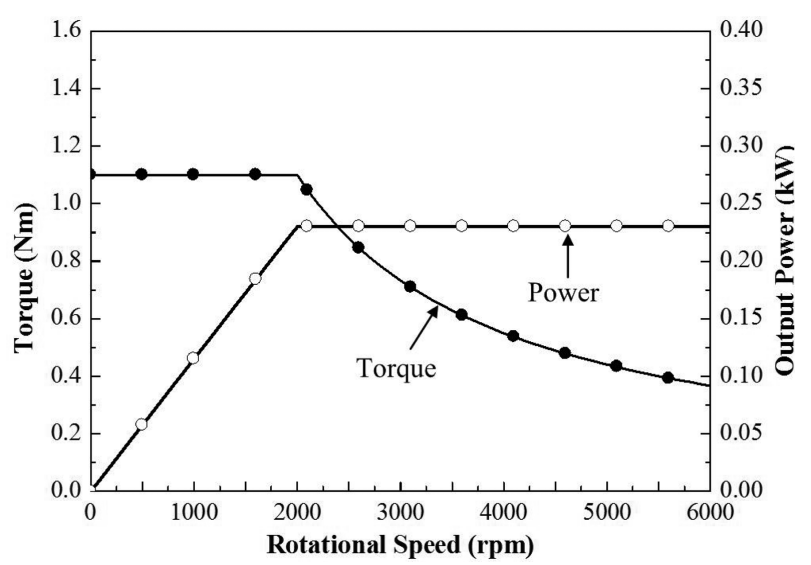

Fig. 7. Torque and output power versus rotational speed of IPMSM in motoring mode.

rotational speed is presented while the generating characteristics according to load conditions are presented in Fig.
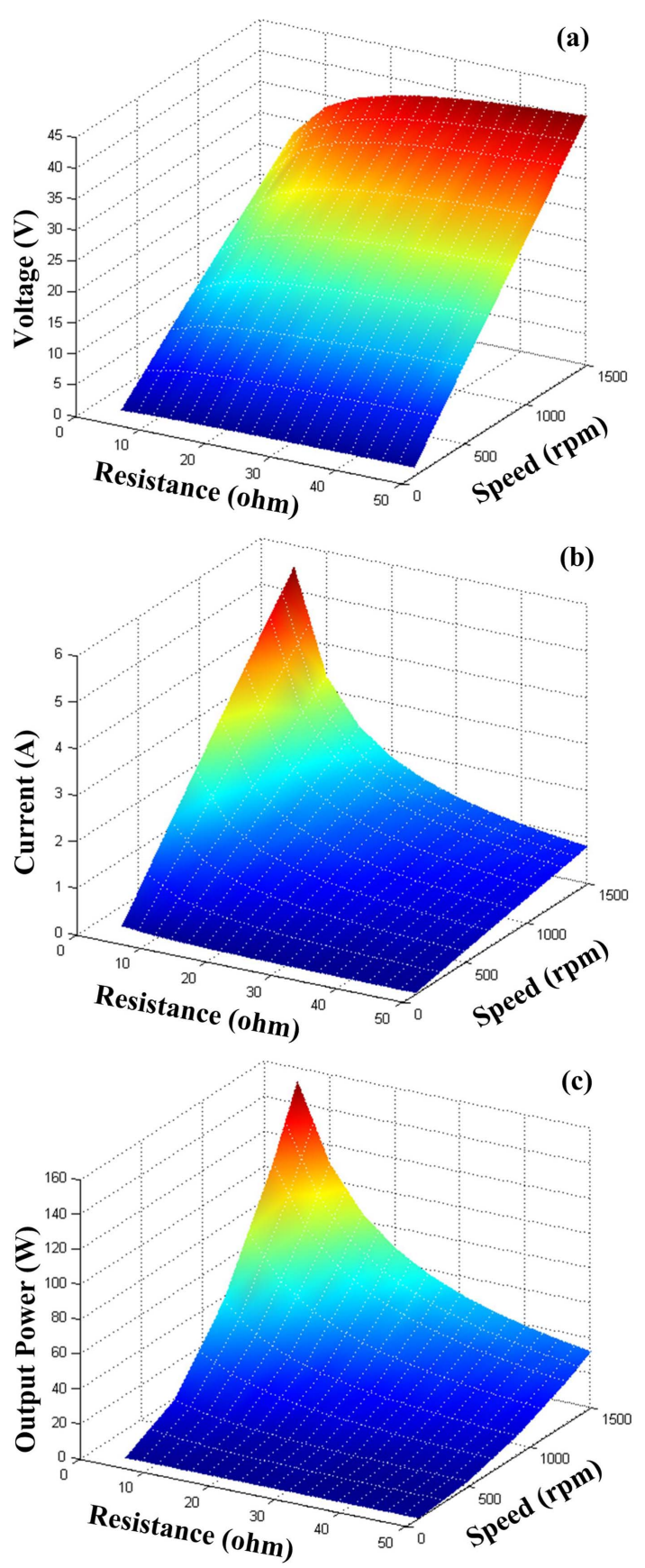

Fig. 8. (Color online) Output characteristics versus load resistance and rotational speed of IPMSM in generating mode.

8. The analyzed results are referred to the experimental set-up construction.

\section{Influence of Phase Current on IPMSM Performance owing to Mechanical Energy Storage}

3.1. Operation of Mechanical Energy Storage System As mentioned before, the DC power from the gene- 

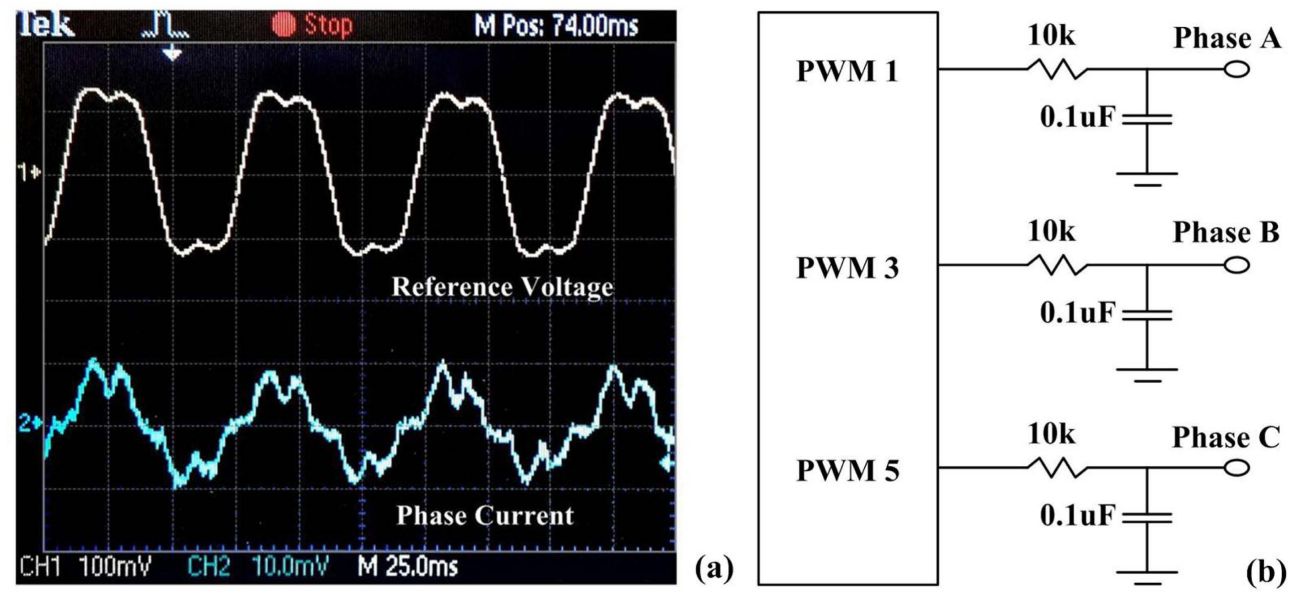

Fig. 9. (Color online) Experimentally measured phase current of SPMSM with mechanical energy storage : (a) reference voltage signal of SVPWM inverter and phase current, (b) RC filter construction for reference voltage.

rating mode is converted to $\mathrm{AC}$ power to operate the MESS through the SVPWM inverter. Since the SPMSM in the MESS is directly connected to the heavy wheel, it can store the mechanical energy with its rotation. The phase current of the SPMSM is controlled by the SVPWM inverter, and Fig. 9(a) presents the reference voltage and measured phase current. Here, the reference voltage is the RC-filtered PWM signal as shown in Fig. 9(b). In this study, the influence of the harmonics of the SPMSM on the power loss characteristics of the IPM machine is not dealt with.

\subsection{Phase Current Characteristics of IPMSM}

As mentioned above, when the SPMSM with the heavy wheel starts rotating, the IPMSM is operated in the MESS load condition. As the speed of SPMSM increases, the current in the IPMSM also increases, resulting in electromagnetic losses, such as copper loss, core loss, and rotor loss. In particular, from the perspective of the IPMSM, the load is a DC load condition owing to the rectifier included in the AC-DC-DC converter, whereas the phase current of IPMSM contains harmonic components. The harmonic components can have a significant influence on the electromagnetic characteristics of the IPMSM; hence, the current is measured in the investigation.

Figure 10 presents the measured current of the IPMSM while the mechanical energy is stored by the SPMSM and the heavy wheel at rotational speed of 333(rpm). The shape of the presented phase current is significantly different from the sinusoidal sine waveform. Owing to the diode rectifier and motor parameters, the current contains harmonic components, which can be separated using Fast Fourier Transform (FFT) as presented in Fig. 11. As it is evident from the result, the phase current of the IPMSM

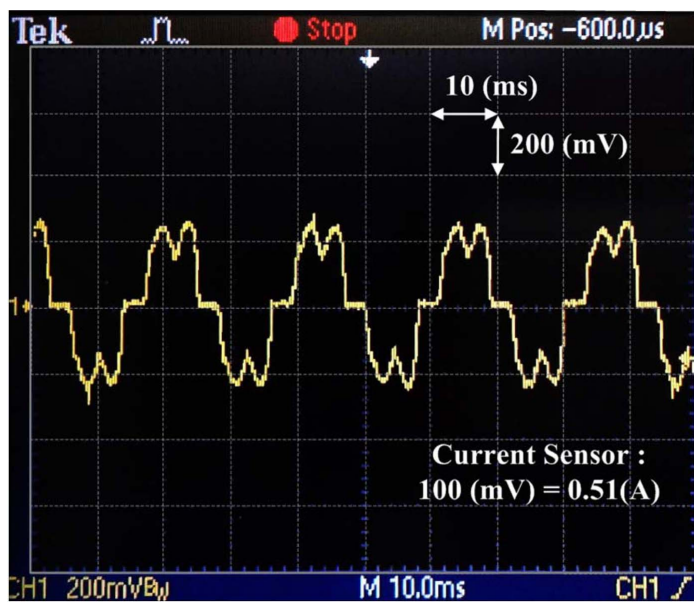

Fig. 10. (Color online) Measured phase current of IPMSM with mechanical energy storage $(910 \mathrm{rpm})$.

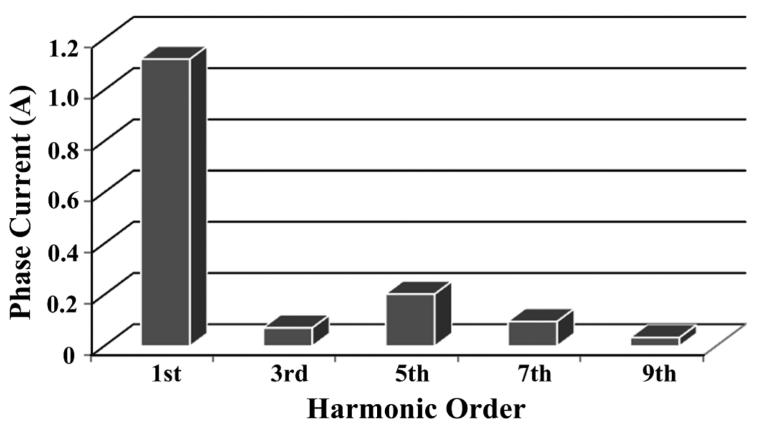

Fig. 11. FFT results of measured current of IPMSM presented in Fig. 7.

owing to the mechanical energy storage contains a high value at the fifth harmonic component. As the authors of [8] reported in their paper, the fifth harmonic component has a significant influence on the electromagnetic power 
losses, such as the core loss and the rotor loss, resulting in the decrease of the machine efficiency.

\subsection{Magnetic Behavior influenced by Measured Cur- rent}

Based on the aforementioned measured phase current of the IPMSM, the magnetic behavior owing to the mechanical energy storage is investigated. The conditions are divided into two categories for a comparative analysis. The first is a no-load condition, wherein the IPMSM is not influenced by the phase current; whereas the other is the MESS load condition, wherein the IPMSM is highly influenced by the mechanical energy storage. Based on the FEM, the radial and tangential components of the magnetic flux density are analyzed. One of the results illustrated in Fig. 12 is obtained at the indicated red point of the stator core in Fig. 1. As evident from the figure, the current, which consists of various harmonics, has a significant influence on the magnetic behavior. Compared to the no-load condition, the magnetic flux density is

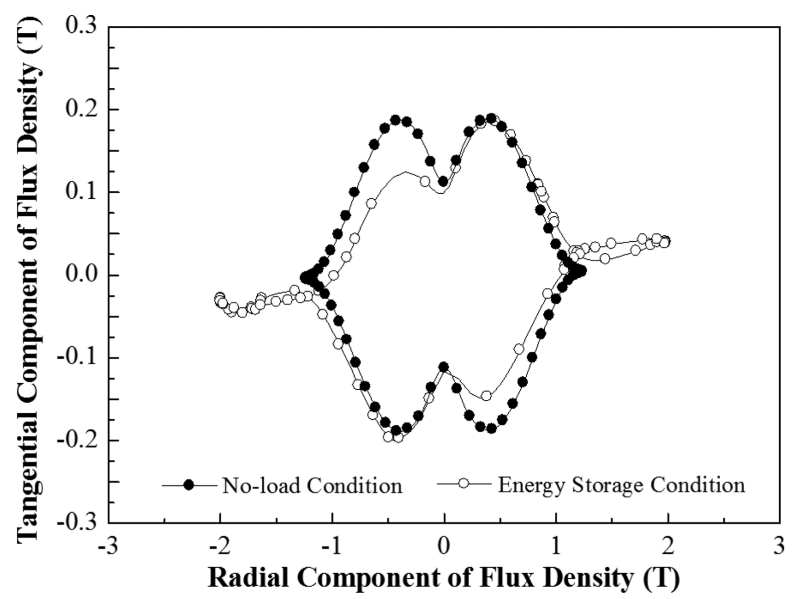

Fig. 12. Radial component and tangential component of flux density according to load conditions.

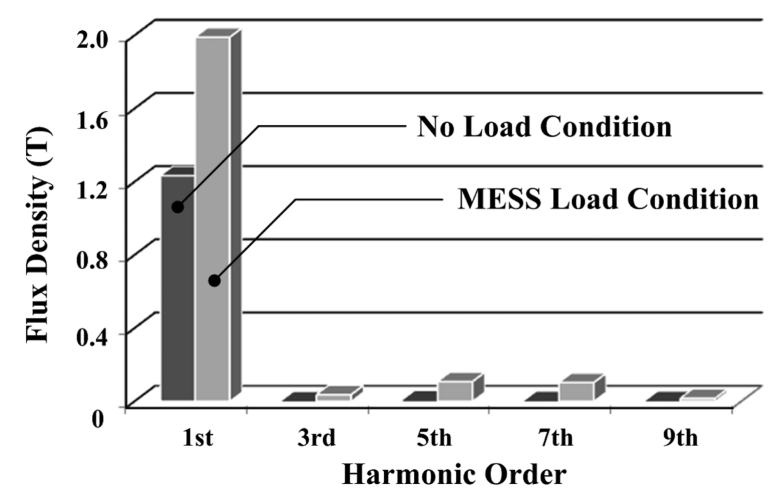

Fig. 13. FFT results of radial component of flux density according to load conditions.

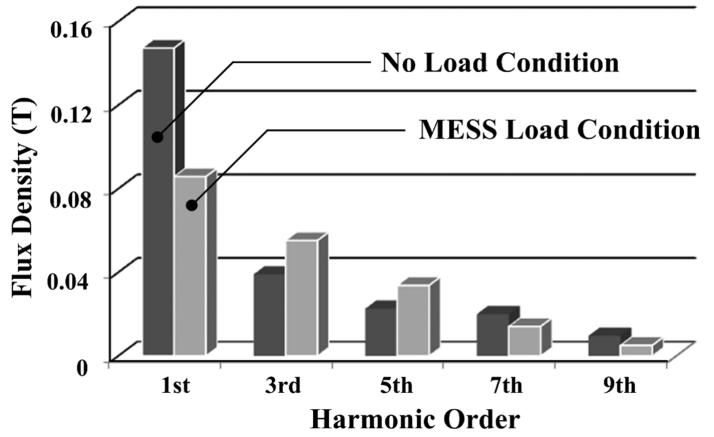

Fig. 14. FFT results of tangential component of flux density according to load conditions.

largely distorted, and this phenomenon is directly related to the electromagnetic power loss characteristics, such as the core loss and the rotor loss of the machine. For a better understanding, Fig. 13 and Fig. 14 present a comparison of the FFT results of the magnetic flux density illustrated in Fig. 10. As shown in Fig. 11, in the case of the radial component of the flux density, owing to the MESS load condition, the fundamental component is increased lightly more than the other harmonic orders. On the other hand, as illustrated in Fig. 14, the fundamental component is reduced owing to the MESS condition, whereas the third and fifth harmonic components are increased. As presented in the study in [9], the radial force distribution can be obtained by the radial and the tangential components of the flux density derived above. Fig. 15 presents the radial force distribution at the point of the stator core based on the obtained flux density, and it is confirmed to be largely distorted in the MESS load condition. Therefore, it can be concluded that the mechanical energy storage causes the electromagnetic vibration source.

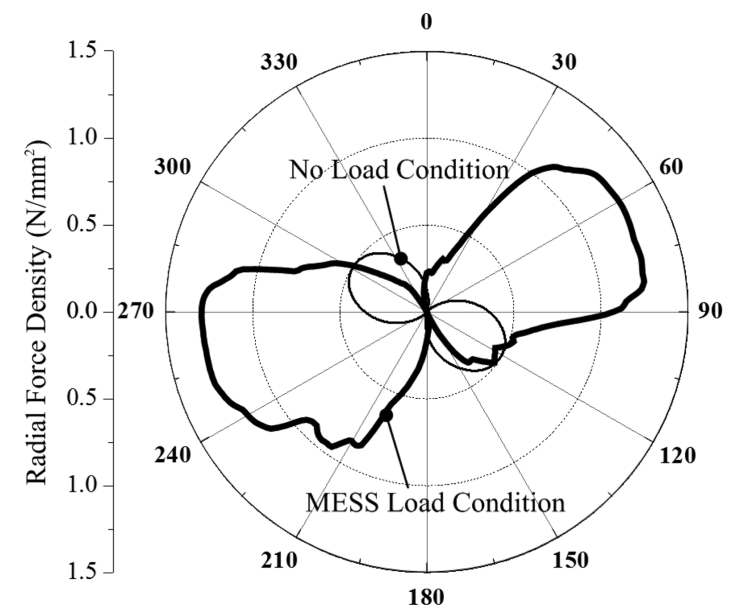

Fig. 15. Radial force distribution derived by radial and tangential component of flux density. 


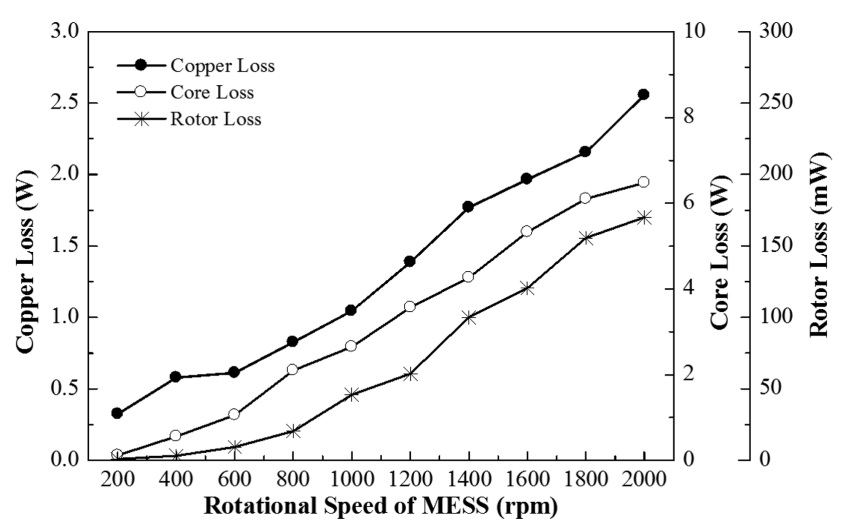

Fig. 16. Power loss characteristics of IPMSM in generating mode while the mechanical energy is being stored : (a) core loss, (b) rotor loss.

\subsection{Power Loss Comparison based on Measured Cur-} rent

The aforementioned harmonic components also have a close relationship with the power loss characteristics. Since the losses are the function of the harmonic components, their value is dramatically increased by the mechanical energy storage system. Based on the electromagnetic field analysis by applying the measured current, the power losses are analyzed, and the related procedure was previously reported in [10]. Consequently, Fig. 16 presents the results of the analysis according to the load conditions, and it can be confirmed that the losses are also increased due to the mechanical energy storage.

\section{Conclusion}

In this paper, the influence of the mechanical energy storage on the IPMSM is investigated by considering the harmonics in the phase current. An experimental set-up was constructed for the investigation, with manufactured machines and power converters, and the electromagnetic characteristics were analyzed. As presented in this study, since the mechanical energy storage system have influence on flux density, radial force and power loss, the solution is required as presented in the previous studies with the improvement of control methodology. Although this paper deals with small-scale machines, the results can be one of the useful references to related potential readers. As a future work, the reduction of the harmonics will be investigated.

\section{Acknowledgment}

This was supported by Korea National University of Transportation in 2017.

\section{References}

[1] P. Zhou, D. Lin, G. Wimmer, N. Lambert, and Z. J. Cendes, IEEE Trans. Magn. 46, 8 (2010).

[2] W. Ren, Q. Xu, Q, Li, and L. Zhou, IEEE Trans. Magn. 52, 7 (2016).

[3] C. Xia, L. Guo, Z. Zhang, T. Shi, and H. Wang, IEEE Trans. Magn. 51, 12 (2015).

[4] H. S. Chen, D. G. Dorrel, and M. C. Tsai, IEEE Trans. Magn. 46, 9 (2010).

[5] J. V. D Veen, L. J. J. Offringa, and A. J. A. Vandenput, IEE Proc.-Electr. Power Appl. 144, 5 (1997).

[6] H. Polinder and M. J. Hoeijmakers, IEE Proc.-Electr. Power Appl. 146, 3 (1999).

[7] Y. Huang, J. Dong, L. Jin, J. Zhu, and Y. Guo, IEEE Trans. Magn. 47, 10 (2011).

[8] S. M. Jang, H. K. Kim, J. Y. Choi, and K. J. Ko, J. Appl. Phys. 105, 7 (2009).

[9] T. Sun, J. M. Kim, G. H. Lee, J. P. Hong, and M. R. Choi, IEEE Trans. Magn. 47, 5 (2010).

[10] Y. S. Park, M. M. Koo, S. M. Jang, H. I. Park, and J. Y. Choi, IEEE Trans. Energy Convers. 28, 4 (2013). 